Article

\title{
Evolutionary Implications of a Peroxidase with High Affinity for Cinnamyl Alcohols from Physcomitrium patens, a Non-Vascular Plant
}

\author{
Teresa Martínez-Cortés ${ }^{1}$ (D) Federico Pomar ${ }^{1}$ and Esther Novo-Uzal ${ }^{2, *(D)}$ \\ 1 Grupo de Investigación en Biología Evolutiva, Centro de Investigaciones Científicas Avanzadas, \\ Universidade da Coruña, 15071 A Coruña, Spain; teresa.martinez.cortes@gmail.com (T.M.-C.); \\ federico.pomar@udc.es (F.P.) \\ 2 Instituto Gulbenkian de Ciência, 2780-156 Oeiras, Portugal \\ * Correspondence: esther.novo.uzal@gmail.com
}

check for updates

Citation: Martínez-Cortés, T.; Pomar F.; Novo-Uzal, E. Evolutionary Implications of a Peroxidase with High Affinity for Cinnamyl Alcohols from Physcomitrium patens,

a Non-Vascular Plant. Plants 2021, 10, 1476. https://doi.org/10.3390/ plants10071476

Academic Editors: Penélope

García-Angulo and

Asier Largo-Gosens

Received: 20 June 2021

Accepted: 15 July 2021

Published: 19 July 2021

Publisher's Note: MDPI stays neutral with regard to jurisdictional claims in published maps and institutional affiliations.

Copyright: (c) 2021 by the authors. Licensee MDPI, Basel, Switzerland. This article is an open access article distributed under the terms and conditions of the Creative Commons Attribution (CC BY) license (https:/ / creativecommons.org/licenses/by/ $4.0 /)$.

\begin{abstract}
Physcomitrium (Physcomitrella) patens is a bryophyte highly tolerant to different stresses, allowing survival when water supply is a limiting factor. This moss lacks a true vascular system, but it has evolved a primitive water-conducting system that contains lignin-like polyphenols. By means of a three-step protocol, including ammonium sulfate precipitation, adsorption chromatography on phenyl Sepharose and cationic exchange chromatography on SP Sepharose, we were able to purify and further characterize a novel class III peroxidase, PpaPrx19, upregulated upon salt and $\mathrm{H}_{2} \mathrm{O}_{2}$ treatments. This peroxidase, of a strongly basic nature, shows surprising homology to angiosperm peroxidases related to lignification, despite the lack of true lignins in P. patens cell walls. Moreover, PpaPrx19 shows catalytic and kinetic properties typical of angiosperm peroxidases involved in oxidation of monolignols, being able to efficiently use hydroxycinnamyl alcohols as substrates. Our results pinpoint the presence in P. patens of peroxidases that fulfill the requirements to be involved in the last step of lignin biosynthesis, predating the appearance of true lignin.
\end{abstract}

Keywords: Physcomitrella; hydroxycinnamyl alcohols; plant evolution; peroxidase; abiotic stress

\section{Introduction}

Land colonization by plants and their subsequent diversification was one of the most important events in the history of life. Terrestrialization forced plants to cope with new stresses absent in the aquatic medium, such as UV light and limited water supply. To avoid water loss, plants developed different strategies to accumulate water in their tissues, to supply it or to minimize its loss. These first land plants, such as mosses, were poikilohydric, whose water potential was equilibrated with surrounding water sources [1]. Likewise, the first evolutionary radiation among land plants is related to the diversification of tracheids, which appeared in vascular plants (tracheophytes), about 450 million years ago and they have been defined as single-celled conduits with lignin in their cell wall [2]. Lignin is a polyphenolic polymer that confers structural support and flexural stiffness to the aerial part of the plant and provides water impermeability, including resistance against tensile forces of water columns. Lignins are mainly formed from the oxidative coupling of three p-hydroxycinnamyl alcohols: p-coumaryl, coniferyl and sinapyl alcohols (monolignols). The cross-coupling reaction of monolignol radicals produces a hydrophobic heteropolymer composed of $p$-hydroxyphenyl (H), guaiacyl (G) and syringyl (S) units [3].

Although lignin has traditionally been linked to vascular plants, polyphenols and lignin-like compounds have been found in species without a true vascular system, such as charophycean green algae [4] and bryophytes [5,6]. Lignin-like compounds are polyphenolic polymers usually detectable with typical methods of lignin determination, such as acetyl bromide or nitrobenzene oxidation, but unlike lignin, they lack $\beta-O-4$ bonds and aryl-glycerol- $\beta$-aryl ether structures. The composition is very variable and in many cases 
unknown but not related to the three $p$-hydroxycinnamyl alcohols that are considered markers for lignins. This finding implies that at least part of the phenylpropanoid pathway that eventually led to lignin biosynthesis was present in algae, and the presence of lignin in tracheids may only have involved the expression of those genes in a different type of cell [7].

The last step of lignin biosynthesis, the oxidation of monolignols, is driven by laccases [8] and peroxidases [9]. Secretory plant peroxidases (class III Prx) are heme-containing glycoproteins that oxidize diverse substrates using hydrogen peroxide as an electron donor. Peroxidases are usually rich in isoenzymes, generated from post-transcriptional and posttranslational modifications [10], with expression patterns usually dependent on development and stress conditions, which make it difficult to assign specific functions to individual peroxidase isoenzymes. Nonetheless, diverse responses to a plethora of stresses or growth conditions have been reported, especially in Arabidopsis, indicating specific functions for the different isoforms $[9,11]$.

Physcomitrium (Physcomitrella) patens is a bryophyte used as a model organism for evolutionary developmental biology and non-vascular plant studies. P. patens shows high tolerance to different environmental cues, such as drought and osmotic and saline stresses, which allows survival in periods when water supply is a limiting factor [12]. RedoxiBase reports 53 class III peroxidases and four pseudogenes in the P. patens genome. However, information about $P$. patens peroxidase functions is scarce. The best characterized peroxidase is Prx34 (PpaPrx13 according to RedoxiBase nomenclature), which was reported to play a role upon fungal attack and catalyze ROS production [13,14].

In this paper, we report the purification of one P. patens peroxidase, upregulated upon salt and oxidative stresses. This enzyme was further purified and characterized, showing homology to angiosperm peroxidases involved in lignification, and with a catalytic efficiency against coniferyl alcohol, a precursor of lignin, of the same order as angiosperm lignification-related peroxidases, despite the fact that $P$. patens does not contain lignin in its cell walls.

\section{Results}

\subsection{Abiotic Stress Strongly Modulates the Expression of a Basic Peroxidase in P. patens}

P. patens is a moss that is highly resistant to abiotic stress, compared to other model plants such as Arabidopsis thaliana, and is especially tolerant to desiccation [15], in line with its phylogenetic position as a moss, and whose ancestors were early colonists of land around 500 million years ago. This bryophyte is thus a useful tool to study responses to abiotic stress. Peroxidases are enzymes known to change their expression pattern in response to different types of stress $[16,17]$. Here, we selected different abiotic stresses and monitored in a time course both peroxidase activity and isoform pattern from protein extracts of P. patens gametophores grown in liquid medium. The results (Figure 1A) show that $\mathrm{H}_{2} \mathrm{O}_{2}$ caused an early increase in peroxidase activity, peaking $1 \mathrm{~h}$ after treatment. The addition of ascorbic acid, a known $\mathrm{H}_{2} \mathrm{O}_{2}$ scavenger, returned peroxidase activity to control levels. $\mathrm{NaCl}$ and salicylic acid (SA) also enhanced peroxidase activity $24 \mathrm{~h}$ after treatment, going back to control levels afterwards. Mannitol slightly changed peroxidase activity throughout the treatment. Although both osmotic and salt stress can be abscisic acid (ABA) dependent, P. patens peroxidase activity in response to ABA treatment did not mirror mannitol or salt stress responses, but strongly decreased from $8 \mathrm{~h}$ after the hormone addition. Moreover, SA and mannitol led to the disappearance of a strongly basic peroxidase isoform, which was instead induced by $\mathrm{H}_{2} \mathrm{O}_{2}$ and $\mathrm{NaCl}$ (Figure $1 \mathrm{~B}$, arrow). We quantified free phenolics as a proxy to measure the stress caused by the different treatments. $\mathrm{H}_{2} \mathrm{O}_{2}$, salt and mannitol treatments significantly enhanced the amount of total phenols in P. patens gametophores (Figure 1C). Based on these results, we pursued the purification and further characterization of a strongly basic peroxidase that was induced by $\mathrm{H}_{2} \mathrm{O}_{2}$ and salt, two major stresses faced by the first plants that colonized land. 
A

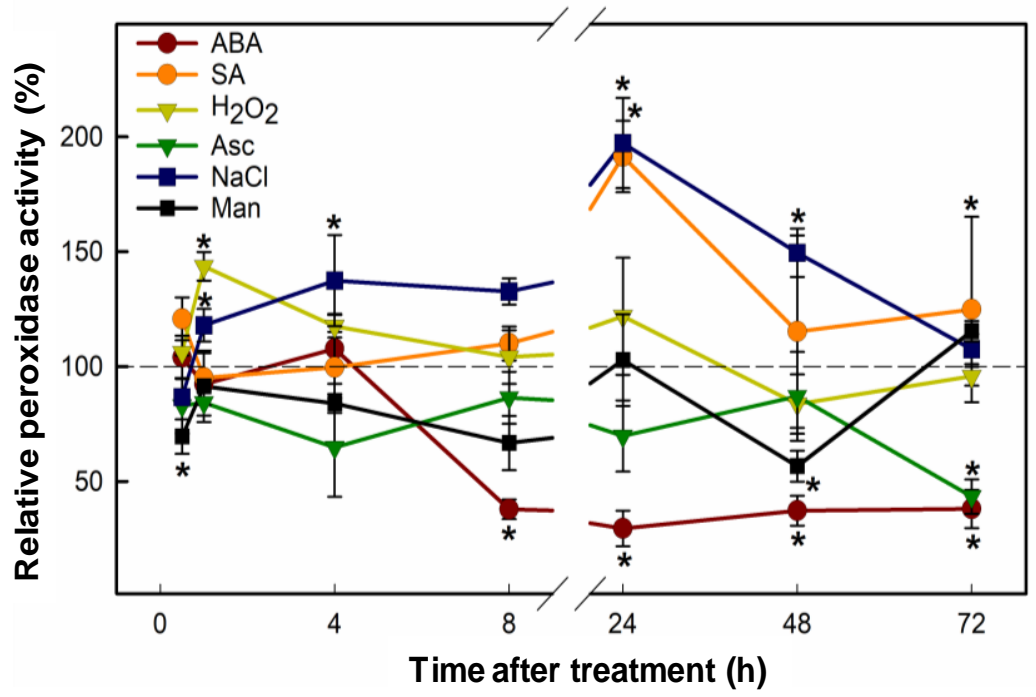

B

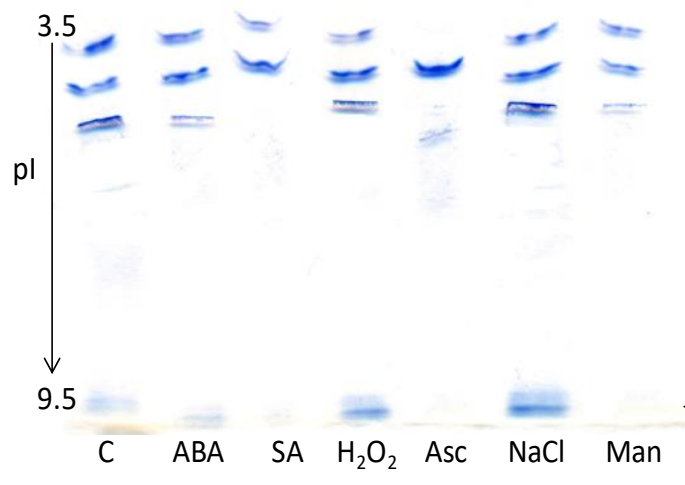

C

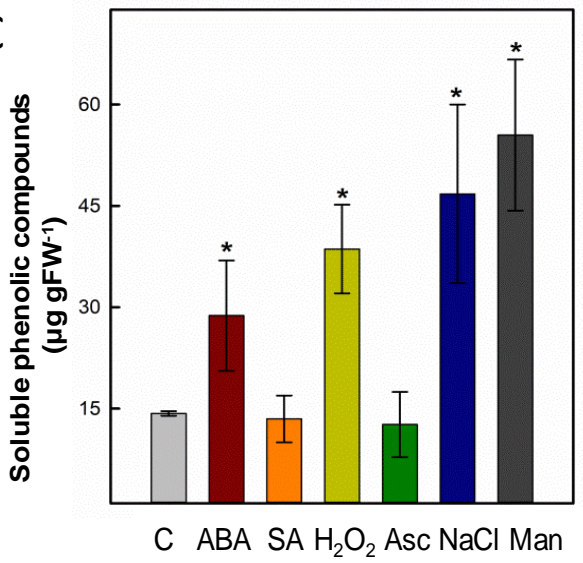

Figure 1. Salt and oxidative stress upregulate the expression of a basic peroxidase from P. patens. (A) Time-course determination of peroxidase activity extracted from $P$. patens gametophores that were subjected to different stress treatments: $\mathrm{H}_{2} \mathrm{O}_{2}$ with or without ascorbic acid (Asc), salt, mannitol (Man), abscisic acid (ABA) and salicylic acid (SA). TMB was used as substrate and peroxidase activity was normalized to control conditions at each time point (shown as a dashed line). (B) Effect at $24 \mathrm{~h}$ of different treatments on peroxidase isoenzyme pattern revealed by IEF stained with 4-MN. C, control; pI, isoelectric point. (C) Effect of stress treatments at $96 \mathrm{~h}$ on soluble phenolic content in P. patens cultures. Data presented are average values \pm SD of $n=3$ experiments. Statistical analysis was carried out with Duncan's test, asterisks represent statistical differences from control $(p<0.05)$.

\subsection{PpaPrx19 Is a $36 \mathrm{kDa}$ Basic Peroxidase}

We extracted total protein from P. patens gametophores grown in control conditions and we then followed a three-step protocol for purification, including ammonium sulfate precipitation, hydrophobic chromatography on phenyl Sepharose and cationic chromatography on SP Sepharose. A fractionated precipitation with ammonium sulfate did not allow the partial purification of the peroxidases of interest, which led us to consider just one fraction, precipitating the proteins with $95 \%\left(\mathrm{NH}_{4}\right)_{2} \mathrm{SO}_{4}$. The fraction was pooled into phenyl Sepharose chromatography, obtaining two major fractions of peroxidases (Figure 2A). The first eluted fraction (F1) contained only acidic peroxidases, while F2 contained both neutral and basic peroxidases and therefore was selected to continue the purification process (Figure 2). After this step, the specific activity for F2 reached $266 \mathrm{nkat} \mathrm{mg}^{-1}$ protein (Table 1). The F2 was then loaded into a cationic exchange chromatography, and the peroxidase bound to SP Sepharose matrix was eluted with a linear gradient of 9.5-11.5 pH. Neutral peroxidases were not retained in the matrix and only one peak of peroxidase activity was eluted, at a pH of 10.9 (Figure 2A). The fraction arising from cationic chromatography migrated as 
two different bands of 36 and $46 \mathrm{kDa}$ in SDS-PAGE electrophoresis, but an IEF showed only one peroxidase with the $\mathrm{pI}$ value determined to be 10.04 (Figure 2B). The peptide mass fingerprinting of the two resultant proteins enabled us to detect that the protein of $46 \mathrm{kDa}$ corresponded to a lipase (accession number XP_001755452). The $36 \mathrm{kDa}$ protein was identified as a predicted protein (access number XP_001781554) which corresponded to PpaPrx19 (according to RedoxiBase nomenclature).
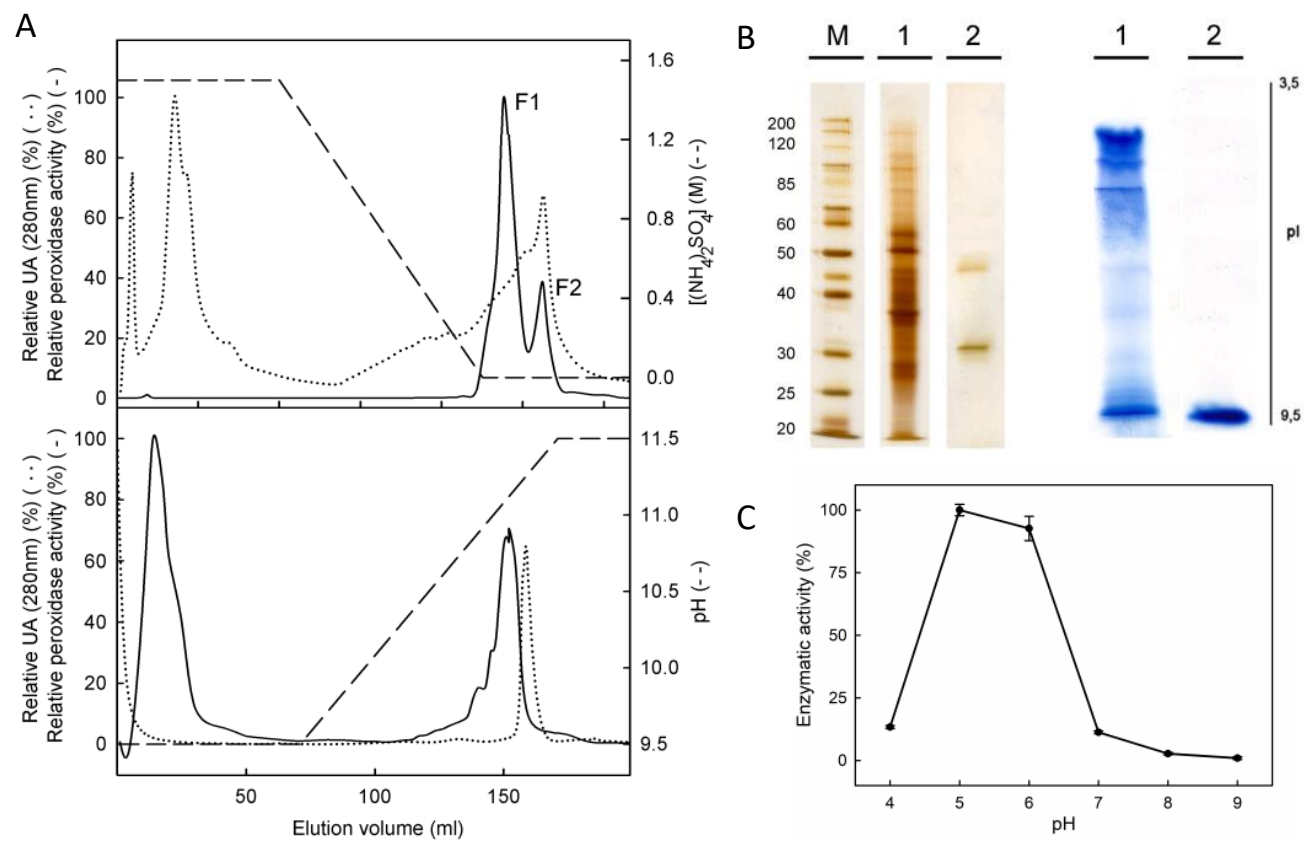

Figure 2. Purified PpaPrx19 is a strongly basic peroxidase. (A) Purification process of PpaPrx19, including adsorption chromatography on phenyl Sepharose (upper panel, peak F2) and cationic exchange chromatography on SP Sepharose (lower panel). Profiles of peroxidase activity and protein are denoted either by a continuous or by a dotted line, respectively. (B) Protein fingerprint in SDSPAGE (left) and peroxidase isoenzyme pattern in IEF (right) of the crude extract (1) and the purified peroxidase (2) SDS-PAGE and IEF were revealed using the silver staining method and 4-MN in the presence of $\mathrm{H}_{2} \mathrm{O}_{2}$, respectively. (C) Dependence on $\mathrm{pH}$ of the purified PpaPrx19 activity. Data presented are average values $\pm \mathrm{SD}$ of $n=3$ experiments.

Table 1. Purification of basic peroxidase PpaPrx19 from P. patens. Peroxidase activity was measured using TMB as substrate.

\begin{tabular}{ccccc}
\hline & $\begin{array}{c}\text { Peroxidase } \\
\text { Activity } \\
\text { (nkat) }\end{array}$ & $\begin{array}{c}\text { Specific } \\
\text { Activity } \\
\text { (nkat mg }{ }^{-1} \text { Protein) }\end{array}$ & $\begin{array}{c}\text { Purification } \\
\text { Fold }\end{array}$ & $\begin{array}{c}\text { Yield } \\
\text { (\%) }\end{array}$ \\
\hline $95 \%\left(\mathrm{NH}_{4}\right)_{2} \mathrm{SO}_{4}$ precipitation & 698 & 28 & 1 & 100 \\
Phenyl Sepharose chromatography & 483 & 266 & 10 & 69 \\
SP Sepharose chromatography & 218 & 23,644 & 850 & 31 \\
\hline
\end{tabular}

We confirmed by RT-qPCR that PpaPrx19 was strongly induced after a treatment with $\mathrm{NaCl}$ and that gene expression was modulated in response to other stresses such as hydrogen peroxide and mannitol (Figure S1).

We also evaluated the dependence on $\mathrm{pH}$ of PpaPrx19 enzymatic activity, using a different $\mathrm{pH}$ (4.0 to 9.0) in the reaction mixture. The purified peroxidase showed the highest activity at $\mathrm{pH} 5.0$, but it rapidly decreased at $\mathrm{pH}>6$ and showed no activity at $\mathrm{pH}$ above 7.0 (Figure 2C). These results do not differ from other peroxidases purified from different sources, with the optimum $\mathrm{pH}$ between 4.5 and 6.5 [18-20]. $\mathrm{pH}$ is critical for peroxidase 
activity because $\mathrm{pH}$ values outside the optimum prevent the heme from binding to the active site of the enzyme [21].

\subsection{PpaPrx19 Is Homologous to Peroxidases with a Role in Lignification}

PpaPrx19 is 332 amino acids long, including a 26 aa N-terminal signal peptide, and it is targeted to the secretory pathway according to analysis with SIGNALP [22] and TARGETP [23] programs. The exon-intron pattern of PpaPrx19 is the second most abundant for P. patens and classic for class III peroxidases, consisting in three exons and two introns [13]. In a BLAST search, PpaPrx19 showed the highest identity at the protein level with two other P. patens peroxidases (PpaPrx18 and PpaPrx09) and the moss Tortula ruralis (Table 2). The rest of the listed peroxidases belong to gymnosperms and angiosperms, and show identity values below $50 \%$, emphasizing the evolutionary distance among them and pointing out the unique characteristics of this peroxidase, at the amino acid level.

Table 2. Comparison of PpaPrx19 mature protein sequence with other class III peroxidases. The most similar peroxidase sequences based on BLASTP searches against the RedoxiBase database were used.

\begin{tabular}{|c|c|c|c|c|}
\hline Prx Name & Species & Taxonomical Group & Identity (\%) & E-Value \\
\hline PpaPrx18 & Physcomitrium patens & Bryophyte & 66 & $3 \times 10^{-144}$ \\
\hline TruPrx01 & Tortula ruralis (star moss) & Bryophyte & 65 & $3 \times 10^{-133}$ \\
\hline PpaPrx09 & Physcomitrium patens & Bryophyte & 61 & $1 \times 10^{-124}$ \\
\hline CppPrx02 & Citrus $x$ Paradisi $x$ Poncirus & Angiosperm & 49 & $3 \times 10^{-97}$ \\
\hline PtaPrx102 & Pinus taeda (loblolly pine) & Gymnosperm & 50 & $8 \times 10^{-97}$ \\
\hline CsPrx62 & Citrus sinensis & Angiosperm & 49 & $2 \times 10^{-96}$ \\
\hline PabPrx05 & Picea abies (Norway spruce) & Gymnosperm & 49 & $6 \times 10^{-95}$ \\
\hline TsPrx15 & Thellungiella salsuginea & Angiosperm & 47 & $1 \times 10^{-94}$ \\
\hline BrPrx15-1 & Brassica rapa & Angiosperm & 48 & $6 \times 10^{-94}$ \\
\hline PtaPrx28 & Pinus taeda (loblolly pine) & Gymnosperm & 46 & $9 \times 10^{-94}$ \\
\hline PabPrx125 & Picea abies (Norway spruce) & Gymnosperm & 48 & $4 \times 10^{-93}$ \\
\hline GbPrx04 & Ginkgo biloba & Gymnosperm & 49 & $6 \times 10^{-93}$ \\
\hline
\end{tabular}

Given these low identity values, we blasted PpaPrx19 against Arabidopsis peroxidases, in order to infer a putative function. Most of the peroxidases with the highest identity level have a reported role in lignification (Table 3). This was a surprising result, given that $P$. patens has an internal water-conducting system constituted by hydroids and living cells with thick walls [24] containing pre-lignin and lignin-like polyphenols but no true lignin (defined as the polymerized compounds found in vascular plants) has been described [5,6].

Table 3. Reported function of Arabidopsis peroxidases which show highest homology to mature PpaPrx19 protein sequence after a BLAST search with Redoxibase.

\begin{tabular}{cccccc}
\hline Peroxidase Name & TAIR Gene ID & Identity (\%) & E-Value & Function & Reference \\
\hline AtPrx15 & At2g18150 & 47 & $4 \times 10^{-93}$ & Lignification/abiotic stress & [25] \\
AtPrx49 & At4g36430 & 47 & $6 \times 10^{-93}$ & Lignification & [26] \\
AtPrx53 & At5g06720 & 46 & $1 \times 10^{-92}$ & Lignification & {$[27]$} \\
AtPrx14 & At2g18140 & 48 & $2 \times 10^{-92}$ & Biotic stress & {$[28]$} \\
AtPrx22 & At2g38380 & 47 & $5 \times 10^{-91}$ & Cold tolerance & {$[29]$} \\
AtPrx52 & At5g05340 & 48 & $1 \times 10^{-89}$ & Lignification & {$[30]$} \\
AtPrx32 & At3g32980 & 45 & $5 \times 10^{-88}$ & Cell elongation & [31] \\
AtPrx34 & At3g49120 & 45 & $7 \times 10^{-88}$ & Oxidative burst & [32] \\
\hline
\end{tabular}

\subsection{PpaPrx19 Shows High Affinity for Cinnamyl Alcohols}

The homology that PpaPrx19 shows with peroxidases reportedly involved in lignification, together with the reported presence of lignin-like polyphenols in P. patens, led us to characterize this peroxidase based on its preferred substrates, using different well-known 
peroxidase substrates, including natural precursors of lignin monomers (Table 4). Ascorbic acid is a typical substrate for class I (ascorbate) peroxidases but is poorly oxidized by class III secretory peroxidases [33]. The oxidation of NADH by peroxidases has been associated with cell wall loosening [34]. IAA is an in vitro peroxidase substrate and it has been reported to be catalyzed in vivo in relation to cell growth [35]. Hydroxycinnamic acids such as ferulic acid can be incorporated into suberin [36] and ferulate can also lead to cross-linking of the cell wall [37]. Coniferyl and sinapyl alcohols are polymerized by apoplastic peroxidases to form lignin [38]. Results showed that PpaPrx19 is able to use each assayed substrate, except ascorbic acid and $\mathrm{NADH}$, although IAA was a poor substrate for PpaPrx19. This peroxidase is able to oxidize ferulic acid $\left(0.53 \pm 0.07 \mathrm{nkat}_{\mu \mathrm{g}}{ }^{-1}\right.$ protein $)$ and sinapyl alcohol $\left(0.08 \pm 0.01 \mathrm{nkat}^{\mathrm{N}} \mathrm{g}^{-1}\right.$ protein) but the highest activity is shown using

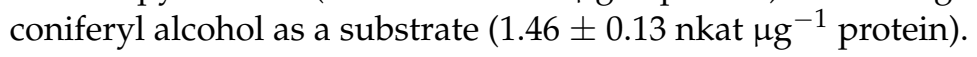

Table 4. Enzymatic activities (nkat $\mu \mathrm{g}^{-1}$ protein) of purified peroxidase PpaPrx19 in the presence of different substrates. Data presented are average values \pm SD of $n=3$ experiments. n.d. not detected.

\begin{tabular}{cc}
\hline Substrate & Peroxidase Activity \\
\hline Ascorbic acid & n.d. \\
NADH & n.d. \\
Indole-3-acetic acid (IAA) & $0.01 \pm 0.00$ \\
Ferulic acid & $0.53 \pm 0.07$ \\
Coniferyl alcohol & $1.46 \pm 0.13$ \\
Sinapyl alcohol & $0.08 \pm 0.01$ \\
\hline
\end{tabular}

Trying to decipher a putative role in cinnamyl alcohol oxidation, we determined the catalytic parameters of PpaPrx19 for coniferyl and sinapyl alcohols. To calculate the kinetic constants, hydrogen peroxide was used at saturation levels $(0.5 \mathrm{mM})$. The $K_{M}$ values were calculated according to Lineweaver-Burk equations. For PpaPrx19, apparent $K_{M}$ values were similar for both alcohols $(16.7 \mu \mathrm{M}$ for coniferyl alcohol and $20.8 \mu \mathrm{M}$ for sinapyl alcohol). However, $K_{\text {cat }}$ is much higher for coniferyl alcohol, rendering a higher catalytic efficiency $\left(K_{\text {cat }} / K_{\mathrm{M}}\right)$, making coniferyl alcohol the best substrate (Table 5).

Table 5. Apparent $K_{\mathrm{M}}, K_{c a t}$ and $K_{c a t} / K_{\mathrm{M}}$ values for coniferyl alcohol (CA) and sinapyl alcohol (SA) shown by PpaPrx19.

\begin{tabular}{cccc}
\hline Substrate & $\boldsymbol{K}_{\mathbf{M}}(\boldsymbol{\mu} \mathbf{M})$ & $\boldsymbol{K}_{\text {cat }}\left(\mathbf{s}^{-\mathbf{1}}\right)$ & $\boldsymbol{K}_{\text {cat }} / \boldsymbol{K}_{\mathbf{M}}\left(\boldsymbol{\mu} \mathbf{M}^{-\mathbf{1}} \mathbf{s}^{-\mathbf{1}}\right)$ \\
\hline CA & 16.7 & 3940.0 & 235.8 \\
SA & 20.8 & 281.0 & 13.5 \\
\hline
\end{tabular}

With these extraordinary biochemical characteristics in mind, we searched for structural determinants that define a particular type of isoenzyme, the syringyl peroxidases [39]. We aligned PpaPrx19 with peroxidases with experimental capacity for oxidizing sinapyl alcohol, including ZePrx (the paradigmatic syringyl peroxidase); ATP A2 and HRP which are unable to oxidize sinapyl alcohol; and the three peroxidases that show the highest identity to PpaPrx19, as shown in Table 2. PpaPrx19 not only contains conserved residues important for catalytic mechanisms and the amino acids required for coordination of two $\mathrm{Ca}^{2+}$ ions (Figure 3), but it also presents most of the structural determinants of syringyl peroxidases (marked in red in Figure 3), which suggests that this peroxidase has no structural restrictions to oxidizing sinapyl alcohol [39]. As a matter of fact, the PpaPrx19 catalytic properties suggest this peroxidase shows a low $K_{\mathrm{M}}$ for sinapyl alcohol and its ability to oxidize this substrate in vitro (Table 5). 


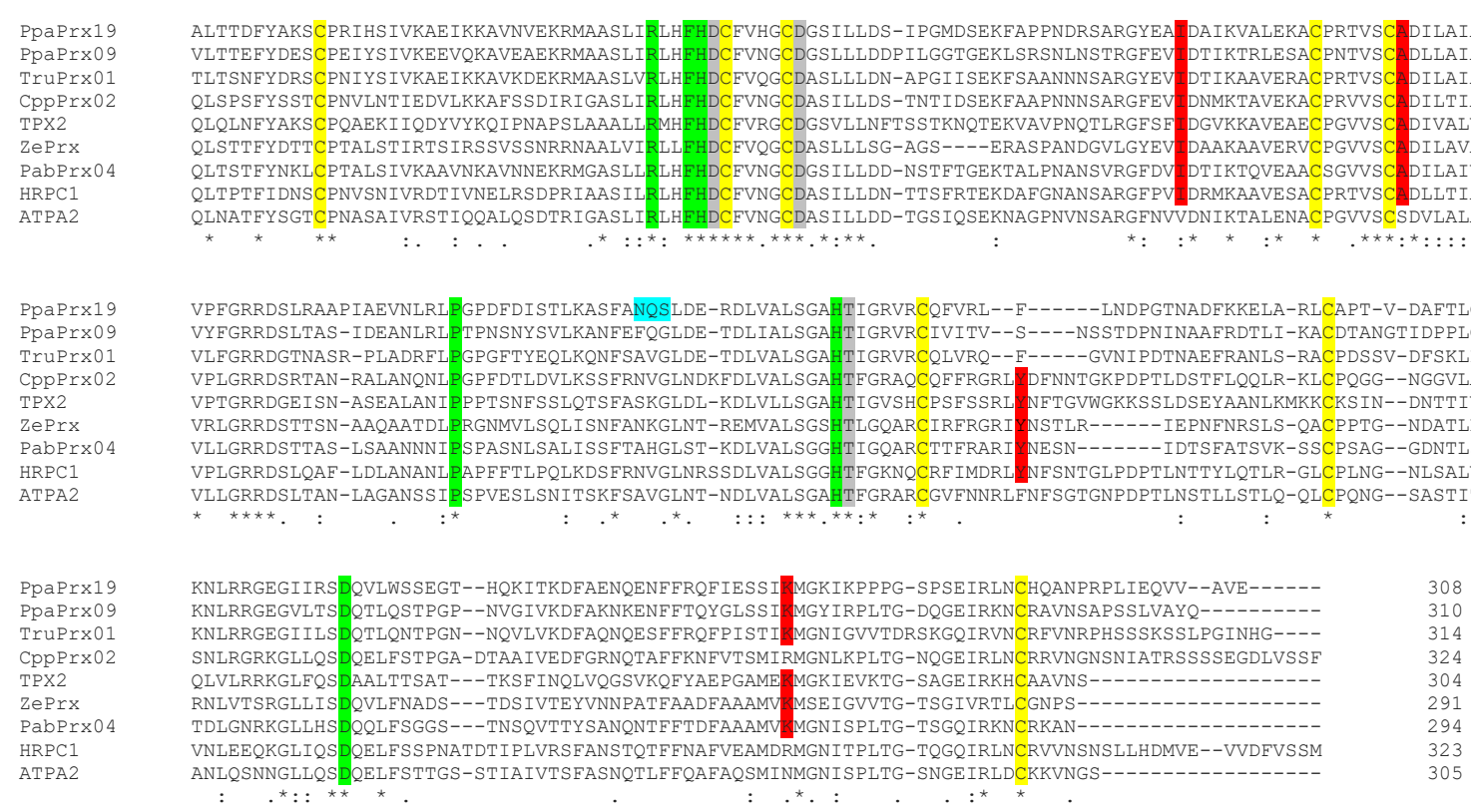

Figure 3. PpaPrx19 shows most of residues characteristic of syringyl peroxidases. Amino acid alignment of mature peroxidase sequences, including those purified in this study (PpaPrx19), Arabidopsis thaliana ATP A2 (CAA68212), horseradish peroxidase HRPC1 (AAA33377), Picea abies PabPrx04 (CAH10839), Zinnia elegans ZePrx (CAI54302), Solanum lycopersicum TPX2 (AAA65636) and the three peroxidases that show the highest identity to PpaPrx19 (PpaPrx09, TruPrx01 and CppPrx02). Conserved residues important for catalytic mechanisms are shaded in green, the calcium-binding sites are shaded in gray, the S-S bridge-forming cysteines are shaded in yellow, putative N-glycosylation site of PpaPrx19 is shaded in blue and structural determinants of syringyl peroxidases are shaded in red. Consensus symbols: ${ }^{\star *}$ indicates fully conserved residues, ' $:$ ' indicates conserved substitutions and '.' indicates semiconserved residue substitutions.

\subsection{PpaPrx19 Associates with Lignin Biosynthesis Enzymes and Cell Wall-Related Proteins}

Finally, we searched for protein associations by means of STRING (string-db.org). This program provides a network of predicted associations for a particular group of proteins based on high-throughput experimental data, literature and database mining [40]. In the case of PpaPrx19, it is located in the center of a network comprising 10 proteins (Figure S2), which are listed as having unknown functions in the poorly annotated P. patens genome. We then searched, for each predicted P. patens protein, its closest homolog in Arabidopsis. The results are shown in Table 6 . The proteins with the highest score are three cinnamyl alcohol dehydrogenases (CADs) and one O-methyl-transferase (OMT). CAD participates in the lignin biosynthetic pathway, catalyzing the conversion of cinnamyl aldehydes into their corresponding alcohols. Unfortunately, the CAD proteins identified by STRING have not been characterized biochemically, although they are known to be expressed in lignifying tissues [41]. The associated OMT has been reported to have high affinity (in the $\mu \mathrm{M}$ range) for a plethora of phenylpropanoids, such as coniferyl alcohol and aldehyde, as well as quercetin [42].

We also performed an analysis in Phytozome (https://phytozome.jgi.doe.gov/pz/ portal.html; accessed on 8 July 2021) and searched for coexpression patterns with PpaPrx19. The list (Table S1) comprises phenylalanine ammonia-lyase (PAL), the first enzyme of phenylpropanoid metabolism, which includes the branch that leads to lignin formation [3]. Other enzyme-encoding genes are also coexpressed with PpaPrx19, such as $\beta-1,3$-glucanase-related and exostosin heparin sulfate glycosyltransferase-related, both associated with remodeling of the cell wall [43]. Moreover, the WRKY transcription factors have been reported to be involved in the regulation of lignin deposition [44]. These associations support the putative involvement of PpaPrx19 in the formation of lignin or lignin-like compounds. 
Table 6. List of proteins PpaPrx19 (PP1S306_37V6.1) has interactions with, based on STRING.

\begin{tabular}{ccccc}
\hline P. patens Protein ID & \multicolumn{2}{c}{ A. thaliana Homolog } & Function & Score \\
\hline PP1S84_209V6.1 & CADG & AT1G72680.1 & Cinnamyl alcohol dehydrogenase & 0.650 \\
PP1S126_185V6.1 & CAD9 & AT4G39330.1 & Cinnamyl alcohol dehydrogenase & 0.650 \\
PP1S163_63V6.1 & ELI3-2 & AT4G37990.1 & Cinnamyl alcohol dehydrogenase & 0.650 \\
PP1S56_71V6.1 & OMT1 & AT5G54160.1 & O-methyltransferase & 0.650 \\
PP1S123_38V6.1 & & AT1G78780 & PR protein & 0.546 \\
PP1S34_74V6.1 & & AT1G29850 & DNA binding \\
PP1S141_102V6.3 & MBF1A & AT2G42680.1 & Multiprotein bridging factor \\
CHI & MBF1B & AT2G43590 & Chitinase & 0.531 \\
PP1S96_94V6.1 & AT3G58680.1 & Multiprotein bridging factor \\
PP1S35_215V6.1 & MBF1B & AT3G58680.1 & Multiprotein bridging factor & 0.521 \\
\hline
\end{tabular}

\section{Discussion}

The cell wall is characteristic of all plant cells, although its composition varies depending on the cell type, the lineage and environmental conditions. Therefore, the plant cell wall is essential for cell development and in responses to stress, being able to plastically adapt to the cell's needs. Several innovations that arose during plant evolution, such as lignin and suberin, help to promote this plasticity. Lignin is thought to have emerged with vascular plants 450 million years ago, but lignin-like or pre-lignin compounds have been detected in bryophytes and algae $[5,45]$. The last step of lignin biosynthesis, the oxidation of hydroxycinnamyl alcohols, is catalyzed by class III peroxidases. In this work, we report the purification of a P. patens peroxidase, PpaPx19, with the ability to oxidize hydroxycinnamyl alcohols (Tables 4 and 5). This characteristic is surprising not just because P. patens does not lignify, but also because of the atypical kinetic properties shown by this peroxidase. In our experiments, PpaPrx19 showed $K_{\mathrm{M}}$ values for hydroxycinnamyl alcohols similar to other peroxidases involved in lignification, from flowering and non-flowering plants. While PpaPrx19 showed $K_{\mathrm{M}}$ values of 16.7 and $20.8 \mu \mathrm{M}$ for coniferyl and sinapyl alcohols, respectively (Table 5), the gymnosperm Picea abies contains two basic peroxidases involved in lignification with reported $K_{\mathrm{M}}$ values for coniferyl alcohol of 16.7 and $23.2 \mu \mathrm{M}$ [46]. In angiosperms, $K_{\mathrm{M}}$ values have been reported for zinnia ( $83 \mu \mathrm{M}$ for coniferyl alcohol) and tomato $(11.4 \mu \mathrm{M}$ for syringaldazine, a chemical analog of sinapyl alcohol) $[47,48]$.

The use of catalytic efficiency $\left(K_{\text {cat }} / K_{\mathrm{M}}\right)$ is preferable in order to compare diverse enzymes and substrates, although very few peroxidase reports calculate this parameter to evaluate enzyme kinetics. In the lycophyte Selaginella, two basic peroxidases show values of 3.55 and $28.63 \mu \mathrm{M}^{-1} \mathrm{~s}^{-1}$ with coniferyl alcohol [20]. GbPrx09 from Ginkgo biloba, a gymnosperm, showed values of $4.91 \mu \mathrm{M}^{-1} \mathrm{~s}^{-1}$ for coniferyl alcohol [17]. In dicots, ZePrx from Z. elegans showed a $K_{\text {cat }} / K_{\mathrm{M}}$ ratio for coniferyl alcohol of $1.20 \mu \mathrm{M}^{-1} \mathrm{~s}^{-1}$ [48] and TPX1 (from tomato) showed a $K_{\text {cat }} / K_{\mathrm{M}}$ for syringaldazine of $1.50 \mu \mathrm{M}^{-1} \mathrm{~s}^{-1}$ [47]. In monocots, PviPRX9 from Panicum virgatum showed a $K_{\text {cat }} / K_{\mathrm{M}}$ ratio for coniferyl alcohol of $1.60 \mu \mathrm{M}^{-1} \mathrm{~s}^{-1}[49]$.

The $K_{c a t} / K_{\mathrm{M}}$ value obtained for PpaPrx19 using coniferyl alcohol as a substrate is not only higher than for peroxidases involved in lignification [48], but also for other enzymes involved in phenylpropanoid metabolism, such as PAL (the first enzyme of the route, [50]), CCR (the first committed enzyme of lignin biosynthesis, [51]) and CAld5H, involved in last steps of lignin biosynthesis [52].

Moreover, our results indicate that PpaPrx19 is able to efficiently use sinapyl alcohol as a substrate. While coniferyl alcohol is easily oxidized by most peroxidases, the capacity of these enzymes to oxidize sinapyl alcohol is not such a common fact and defined a new subgroup named syringyl peroxidases [39]. PpaPrx19 has most of the structural determinants of this new subgroup [39] in the protein primary structure (Figure 3, shaded in red). These structural motifs determine the syringyl oxidase activity shown by peroxidases, but are absent in the two paradigmatic G peroxidases, ATP A2 and HRP A2 from Arabidopsis 
and horseradish, respectively. These structural motifs agree with the experimental capacity of PpaPrx19 of oxidizing sinapyl alcohol in vitro.

All these data strongly suggest that the peroxidase PpaPrx19 may have been involved in lignin biosynthesis, if such a pathway was present in P. patens, i.e., PpaPrx19 fulfills the kinetic and structural requirements to oxidize coniferyl alcohol. The presence of an enzyme involved in the biosynthetic route of a compound that appeared later in an evolutionary context is not surprising. Thermospermine emerges as one example of a metabolite typical of vascular plants recently described in non-vascular plants. The only reported function for thermospermine is the regulation of xylem cell maturation, which makes the function it may have in non-vascular plants unclear [53]. It is widely accepted that promiscuous enzymes with several putative substrates are more likely to be recruited to novel metabolic routes [54]. Therefore, a peroxidase with multiple substrates but with particular affinity for coniferyl alcohol would be a good candidate to participate in what eventually would constitute the pathway leading to lignin formation.

Several reports [55-57] indicate that the first appearance of the entire lignin biosynthesis pathway enzymes (excluding the pathway that leads to syringyl lignin formation), from the catalysis of phenylalanine to coniferyl alcohol formation, took place in mosses (P. patens), regardless the fact that $P$. patens does not accumulate lignin in the cell wall. Thus, the P. patens genome has all the genes necessary for the biosynthesis of lignin, and according to the results presented in this paper, at least some of the enzymes are expressed and functional, but the route does not take place and lignin is not polymerized. Nonetheless, a pre-lignin pathway has been recently suggested, revealing a role of caffeate units for the formation of the P. patens cuticle, coupled with ascorbate metabolism [6]. This finding suggests that the biosynthesis of lignin-like or pre-lignin compounds may not originate from the precursors described for canonical lignin and that the enzymes involved in its synthesis have broader specificity than the enzymes participating in true lignin from vascular plants, making lignin evolution an exciting field to explore.

PpaPrx19 may have a function that in vascular plants was later derived for involvement in lignification. This hypothesis is supported by its structural and kinetic homology to peroxidases with an already described role in lignin biosynthesis, such as ZePrx [39,48], and association with other enzymes of the lignin biosynthetic route. The appearance of a primitive water-conducting system, together with stomata and cuticle, were innovations developed by plants during the transition from water to land. Renault [6] already proved the existence of a pre-lignin pathway involved in the formation of the P. patens cuticle. Given all this, although the actual function of PpaPrx19 in P. patens physiology remains unclear and should be further studied, it is likely involved in the remodeling of the cell wall in response to environmental stress, based on this peroxidase ability of oxidizing phenolic compounds and its upregulation upon several conditions related to water deficiency, the paradigmatic stress for poikilohydric plants lacking a true vascular system.

\section{Materials and Methods}

\subsection{Plant Material and Treatments}

P. patens was provided by the Biotechnology Department of the Universidad Politécnica (Madrid, Spain) and cultured as described previously [58]. P. patens gametophores were maintained in solid medium under standard conditions in a growth chamber at $25^{\circ} \mathrm{C}$ with a $16 \mathrm{~h}$ photoperiod. For stress treatments, P. patens gametophores were grown in liquid medium on a rotator shaker (130 rpm) and then transferred into medium supplemented with $5 \mathrm{mM} \mathrm{H}_{2} \mathrm{O}_{2}$ (with or without $10 \mathrm{mM}$ ascorbic acid), $250 \mathrm{mM} \mathrm{NaCl}, 250 \mathrm{mM}$ mannitol, $10 \mu \mathrm{M}$ abscisic acid (ABA) and $1 \mathrm{mM}$ salicylic acid (SA), respectively [59-62].

\subsection{Protein Extraction and Precipitation}

Gametophore samples ( $400 \mathrm{~g}$ ) were homogenized in $50 \mathrm{mM}$ Tris-HCl buffer (pH 7.5) containing $1 \mathrm{mM}$ EDTA, $1 \mathrm{M} \mathrm{KCl}$, and $0.05 \mathrm{~g}$ PVPP per $\mathrm{g}$ of tissue. The homogenate was filtered through nylon layers and centrifuged at $27,000 \times g$ for $30 \mathrm{~min}$ at $4{ }^{\circ} \mathrm{C}$. The 
supernatant was dialyzed on cellulose membranes. After protein precipitation with $95 \%$ saturation ammonium sulfate, the precipitate was resuspended in $50 \mathrm{mM}$ Tris- $\mathrm{HCl} \mathrm{pH} 7.5$ and dialyzed overnight against the same buffer.

\subsection{Purification of PpaPrx19}

Purification of P. patens peroxidase 19 was performed in the AKTA System (GE Healthcare, Barcelona, Spain). The dialyzed sample was concentrated in Amicon ${ }^{\circledR}$ Ultra (Merck Millipore, Barcelona, Spain) and dissolved in $1.5 \mathrm{M}\left(\mathrm{NH}_{4}\right)_{2} \mathrm{SO}_{4}$. In the first step, hydrophobic chromatography, proteins were separated on a phenyl Sepharose ${ }^{\mathrm{TM}} 6$ Fast Flow (GE Healthcare, Barcelona, Spain) $31.5 \times 1.0 \mathrm{~cm}$ gel bed column at a flow rate $1.0 \mathrm{~mL} \cdot \mathrm{min}^{-1}$, and fractions of $5.0 \mathrm{~mL}$ were recovered. The eluent chromatography program was as follows: from 0 to $160 \mathrm{~min}(100 \% \mathrm{~A}, 0 \% \mathrm{~B})$, from 160 to $360 \mathrm{~min}(0 \%$ to $100 \% \mathrm{~B})$ and from 360 to $515 \mathrm{~min}(100 \% \mathrm{~B})$, where buffer A was $50 \mathrm{mM}$ Tris- $\mathrm{HCl}$ (pH 7.5) containing $1.5 \mathrm{M}\left(\mathrm{NH}_{4}\right)_{2} \mathrm{SO}_{4}$, and buffer B was $50 \mathrm{mM}$ Tris- $\mathrm{HCl}(\mathrm{pH} 7.5)$.

The second step involved ion-exchange chromatography on SP Sepharose Fast Flow (GE Healthcare). To do so, the peroxidase-enriched fractions obtained from the hydrophobic chromatography were dialyzed against $50 \mathrm{mM}$ CAPS, ( $\mathrm{pH} 9.5)$, and loaded on a $17.5 \times 1.0 \mathrm{~cm}$ gel bed column equilibrated with $50 \mathrm{mM} \mathrm{CAPS} \mathrm{(pH} \mathrm{9.5),} \mathrm{at} \mathrm{a} \mathrm{flow} \mathrm{rate} \mathrm{of}$

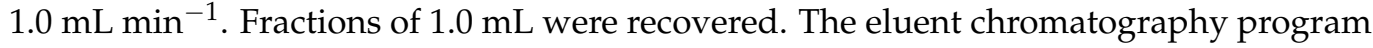
was as follows: from 0 to $70 \mathrm{~min}(100 \% \mathrm{~A}, 0 \% \mathrm{~B})$, from 70 to $170 \mathrm{~min}(0-100 \% \mathrm{~B})$ and from 170 to $330 \mathrm{~min}(100 \% \mathrm{~B})$, where buffer A was $50 \mathrm{mM}$ CAPS (pH 9.5), and buffer B was $50 \mathrm{mM}$ CAPS (pH 11.5).

\subsection{Peroxidase Activity Determination}

Peroxidase activity was determined in a spectrophotometer at $25{ }^{\circ} \mathrm{C}$ in a reaction medium containing $50 \mathrm{mM}$ sodium acetate buffer ( $\mathrm{pH}$ 5.0) and $0.5 \mathrm{mM} \mathrm{H}_{2} \mathrm{O}_{2}$ with tetramethylbenzidine (TMB) $0.1 \mathrm{mg} \cdot \mathrm{mL}^{-1}$ as substrate $\left(\varepsilon_{652}=39.0 \mathrm{mM}^{-1} \cdot \mathrm{cm}^{-1}\right)$.

Peroxidase activity was also calculated using different substrates: $100 \mu \mathrm{M}$ coniferyl alcohol $\left(\varepsilon_{262}=9.75 \mathrm{mM}^{-1} \cdot \mathrm{cm}^{-1}\right), 100 \mu \mathrm{M}$ sinapyl alcohol $\left(\varepsilon_{271}=4.14 \mathrm{mM}^{-1} \cdot \mathrm{cm}^{-1}\right), 50 \mu \mathrm{M}$ ferulic acid $\left(\varepsilon_{310}=16.6 \mathrm{mM}^{-1} \cdot \mathrm{cm}^{-1}\right), 200 \mu \mathrm{M}$ indole-3-acetic acid $\left(\varepsilon_{261}=3.2 \mathrm{mM}^{-1} \cdot \mathrm{cm}^{-1}\right)$, $1 \mathrm{mM}$ ascorbic acid $\left(\varepsilon_{290}=2.8 \mathrm{mM}^{-1} \cdot \mathrm{cm}^{-1}\right)$ and $150 \mu \mathrm{M} \mathrm{NADH}\left(\varepsilon_{340}=6.22 \mathrm{mM}^{-1} \cdot \mathrm{cm}^{-1}\right)$, as described elsewhere $[48,63]$. For measuring peroxidase activity with ascorbic acid, $50 \mathrm{mM}$ phosphate buffer ( $\mathrm{pH} 7.0$ ) was used.

The $\mathrm{pH}$-dependent enzymatic activity was assessed using $50 \mathrm{mM}$ sodium acetate buffer for $\mathrm{pH} 4.0$ to 6.0 and $50 \mathrm{mM}$ Tris- $\mathrm{HCl}$ for $\mathrm{pH} 7.0$ to 9.0, with TMB as substrate.

\subsection{Kinetic Data Analysis}

For determination of apparent $K_{M}, 5-100 \mu \mathrm{M}$ (coniferyl alcohol) and 1-110 $\mu \mathrm{M}$ (sinapyl alcohol) concentrations of each substrate were used in $50 \mathrm{mM}$ sodium acetate buffer $\mathrm{pH}$ 5.0. $K_{\mathrm{M}}$ values were determined from the Lineweaver-Burk equation, a linear transformation of the Michaelis-Menten equation.

\subsection{Electrophoretic Analysis}

Isoelectric focusing was performed on a Pharmacia Multiphor II system using Ampholine PAGplate ( $\mathrm{pH}=3.5-9.5)$ polyacrylamide gels according to the manufacturer's instructions (GE Healthcare). Peroxidase isoenzymes were stained with 4-methoxy- $\alpha$ naphthol (4-MN) in the presence of $\mathrm{H}_{2} \mathrm{O}_{2}$.

SDS-PAGE was performed on $10 \%(w / v)$ polyacrylamide gels using a MiniProtean ${ }^{\circledR} 3$ Cell electrophoresis kit (Bio-Rad Laboratories, Barcelona, Spain) and a pH 8.8 electrophoresis buffer composed of $192 \mathrm{mM}$ glycine and $25 \mathrm{mM}$ Tris containing $0.1 \%$ SDS. SDS-PAGE was performed at $200 \mathrm{~V}$ for $40-45 \mathrm{~min}$ at room temperature. Proteins were stained with a Plus One Silver Staining kit, according to the manufacturer's instructions (GE Healthcare). 


\subsection{Phenolic Compound Extraction and Quantification}

Gametophores grown in liquid culture were harvested $96 \mathrm{~h}$ after treatment, ground in liquid nitrogen and extracted for soluble phenolic content as previously described [17]. The quantitative determination of phenolics was performed using Folin-Ciocalteu reagent with ferulic acid as standard.

\subsection{Molecular Weight MALDI-TOF/TOF}

The purified peroxidase was analyzed in a MALDI-TOF/TOF instrument as previously described [20]. The search for peptide mass fingerprints and tandem MS spectra was performed in the NCBInr database without taxonomy restriction. Mascot scores for all protein identifications were higher than the accepted threshold for significance (at the $p<0.050$ level, positive rate measured to be 0.047 ).

\subsection{Sequence Data Analysis}

The presence of signal peptides was predicted using TargetP (http:/ / www.cbs.dtu. $\mathrm{dk}$ /services/TargetP/, accessed on 24 November 2020), Bacello (http:/ /gpcr2.biocomp. unibo.it/bacello/index.htm, accessed on 24 November 2020) and TargetLoc (http:/ /abi.inf. unituebingen.de/Services/MultiLoc/index_html, accessed on 24 November 2020). pI prediction was carried out using Compute pI/MW tool from ExPASy (http:/ / web.expasy.org/ compute_pi, accessed on 24 November 2020). A search for protein sequences homologies was performed using BLASTP from Redoxibase (http:/ / www.peroxibase.toulouse.inra.fr, accessed on 4 February 2021) and protein alignments were carried out using Clustal Omega (https:/ / www.ebi.ac.uk/Tools/msa/clustalo/, accessed on 4 February 2021). Search for $\mathrm{N}$-glycosylation sites was performed using the NetNGlyc tool (http:/ / www.cbs.dtu.dk/ services/NetNGlyc, accessed on 25 November 2020).

\subsection{Gene Expression Analysis by RT-qPCR}

Total RNA was isolated from gametophores grown in liquid medium, $24 \mathrm{~h}$ after treatment, with Trizol (Invitrogen, Madrid, Spain) essentially as described in [64]. cDNA was synthesized from $200 \mathrm{ng}$ of total RNA using the iScript ${ }^{\mathrm{TM}}$ cDNA Synthesis Kit (Bio Rad Laboratories, Barcelona, Spain). For RT-qPCR, the constitutively expressed $18 \mathrm{~S}$ gene was used as a reference gene (Fwd 5'-GGACCGATAGGTCTGGGTAA-3' and Rev 5'GCAATCCGAAAACTTCACCG-3') and for PpaPrx19 amplification, the primers Fwd $5^{\prime}$ - CTCACCACTGACTTCTACGC-3' and Rev $5^{\prime}$-TGGGATGCTGTCCAAGAGTA- $3^{\prime}$ were used. The PCR reaction contained Bio-Rad 1x iQ SYBR Green Supermix, $0.3 \mu \mathrm{M}$ primer mix and $2.5 \mu \mathrm{g}$ of cDNA for a $50 \mu \mathrm{L}$ final volume. The PCR program comprised a $1 \mathrm{~min}$ denaturation step at $94{ }^{\circ} \mathrm{C}$ followed by 40 cycles of amplification $\left(94{ }^{\circ} \mathrm{C}\right.$ for $30 \mathrm{~s}, 58{ }^{\circ} \mathrm{C}$ for $45 \mathrm{~s}$ and $72{ }^{\circ} \mathrm{C}$ for $1 \mathrm{~min}$ ) and a final elongation step of $6 \mathrm{~min}$ at $72{ }^{\circ} \mathrm{C}$. Bio-Rad Optical System Software 3.0 was used for data analysis, and relative expression values were calculated from the resulting Ct values [65].

\subsection{Network Construction of PpaPrx19}

The Search Tool for the Retrieval of Interacting Genes/Proteins database (STRING v11) was used to construct the network associated with PpaPrx19. The sequence of PpaPrx19 was loaded in the database and STRING generated a protein-protein interaction (PPI) network with proteins that have interactions with the target protein. Interactions were obtaining with medium confidence from curated databases and textmining.

Supplementary Materials: The following are available online at https:/ /www.mdpi.com/article/10 .3390/ plants10071476/s1, Figure S1: Effect of different abiotic stresses on PpaPrx19 gene expression, Figure S2: Graphical representation of the proteins PpaPrx9 (PP1S306_37V6.1) has interactions with. Table S1. List of coexpression patterns obtained for PpaPrx19 in Phytozome (https:/ / phytozome.jgi. doe.gov/pz/portal.html), accessed on 8 July 2021. 
Author Contributions: Conceptualization, F.P., E.N.-U., T.M.-C.; methodology, T.M.-C.; formal analysis, T.M.-C., E.N.-U.; investigation, T.M.-C.; writing-original draft preparation, E.N.-U.; writingreview and editing, T.M.-C., E.N.-U., F.P.; supervision, E.N.-U., F.P.; funding acquisition, E.N.-U., F.P. All authors have read and agreed to the published version of the manuscript.

Funding: This research was funded by Xunta de Galicia (Spain), grant number INCITE 08PXIB103182PR. E.N.-U. holds an FCT postdoctoral fellowship (SFRH/BPD/112587/2015).

Data Availability Statement: The data is contained within the article.

Acknowledgments: P. patens was kindly provided by Alonso Rodríguez-Navarro from the Biotechnology Department of Universidad Politécnica (Madrid, Spain). The proteomic analysis assistance of Jesús Mateos (INIBIC, A Coruña, Spain) is gratefully acknowledged.

Conflicts of Interest: The authors declare no conflict of interest. The funders had no role in the design of the study; in the collection, analyses, or interpretation of data; in the writing of the manuscript, or in the decision to publish the results.

\section{References}

1. Charron, A.J.; Quatrano, R.S. Between a rock and a dry place: The water-stressed moss. Mol. Plant 2009, 2, 478-486. [CrossRef]

2. Kenrick, P.R.; Crane, P.R. The origin and early evolution of plants on land. Nature 1997, 389, 33-39. [CrossRef]

3. Vanholme, R.; De Meester, B.; Ralph, J.; Boerjan, W. Lignin biosynthesis and its integration into metabolism. Curr. Opin. Biotechnol. 2019, 56, 230-239. [CrossRef] [PubMed]

4. Sørensen, I.; Pettolino, F.A.; Bacic, A.; Ralph, J.; Lu, F.; O’Neill, M.A.; Fei, Z.; Rose, J.K.; Domozych, D.S.; Willats, W.G. The charophycean green algae provide insights into the early origins of plant cell walls. Plant J. 2011, 68, 201-211. [CrossRef]

5. Espiñeira, J.M.; Novo-Uzal, E.; Gómez-Ros, L.V.; Carrión, J.S.; Merino, F.; Ros Barceló, A.; Pomar, F. Distribution of lignin monomers and the evolution of lignification among lower plants. Plant Biol. 2011, 13, 59-68. [CrossRef] [PubMed]

6. Renault, H.; Alber, A.; Horst, N.A.; Basilio Lopes, A.; Fich, E.A.; Kriegshauser, L.; Wiedemann, G.; Ullmann, P.; Herrgott, L.; Erhardt, M.; et al. A phenol-enriched cuticle is ancestral to lignin evolution in land plants. Nat. Commun. 2017, 8, 14713. [CrossRef]

7. Weng, J.K.; Chapple, C. The origin and evolution of lignin biosynthesis. N. Phytol. 2010, 187, 273-285. [CrossRef]

8. Berthet, S.; Demont-Caulet, N.; Pollet, B.; Bidzinski, P.; Cézard, L.; Le Bris, P.; Borrega, N.; Hervé, J.; Blondet, E.; Balzergue, S.; et al. Disruption of LACCASE4 and 17 results in tissue-specific alterations to lignification of Arabidopsis thaliana stems. Plant Cell 2011, 23, 1124-1137. [CrossRef]

9. Shigeto, J.; Tsutsumi, Y. Diverse functions and reactions of class III peroxidases. N. Phytol. 2016, 209, 1395-1402. [CrossRef]

10. Welinder, K.G.; Justesen, A.F.; Kjaersgard, I.V.; Jensen, R.B.; Rasmussen, S.K.; Jespersen, H.M.; Duroux, L. Structural diversity and transcription of class III peroxidases from Arabidopsis thaliana. Eur. J. Biochem. 2002, 269, 6063-6081. [CrossRef] [PubMed]

11. Cosio, C.; Dunand, C. Specific functions of individual class III peroxidase genes. J. Exp. Bot. 2009, 60, 391-408. [CrossRef] [PubMed]

12. Rensing, S.A.; Goffinet, B.; Meyberg, R.; Wu, S.-Z.; Bezanilla, M. The Moss Physcomitrium (Physcomitrella) patens: A model organism for non-seed plants. Plant Cell 2020, 32, 1361-1376. [CrossRef]

13. Lehtonen, M.T.; Akita, M.; Kalkkinen, N.; Ahola-Iivarinen, E.; Rönnholm, G.; Somervuo, P.; Thelander, M.; Valkonen, J.P.T. Quickly-released peroxidase of moss in defense against fungal invaders. N. Phytol. 2009, 183, 432-443. [CrossRef] [PubMed]

14. Lehtonen, M.T.; Akita, M.; Frank, W.; Reski, R.; Valkonen, J.P. Involvement of a class III peroxidase and the mitochondrial protein TSPO in oxidative burst upon treatment of moss plants with a fungal elicitor. Mol. Plant Microbe Interact. 2012, 25, 363-371. [CrossRef] [PubMed]

15. Frank, W.; Ratnadewi, D.; Reski, R. Physcomitrella patens is highly tolerant against drought, salt and osmotic stress. Planta 2005, 220, 384-394. [CrossRef]

16. Su, P.; Yan, J.; Li, W.; Wang, L.; Zhao, J.; Ma, X.; Li, A.; Wang, H.; Kong, L. A member of wheat class III peroxidase gene family, TaPRX-2A, enhanced the tolerance of salt stress. BMC Plant Biol. 2020, 20, 392. [CrossRef]

17. Novo-Uzal, E.; Gutierrez, J.; Martinez-Cortes, T.; Pomar, F. Molecular cloning of two novel peroxidases and their response to salt stress and salicylic acid in the living fossil Ginkgo biloba. Ann. Bot. 2014, 114, 923-936. [CrossRef] [PubMed]

18. Mika, A.; Lüthje, S. Properties of guaiacol peroxidase activities isolated from corn root plasma membranes. Plant Physiol. 2003, 132, 1489-1498. [CrossRef] [PubMed]

19. Sergio, L.; Cardinali, A.; Di Paola, A.; Di Venere, D. Biochemical properties of soluble and bound peroxidases from artichoke heads and leaves. Food Technol. Biotechnol. 2009, 47, 32-38.

20. Martinez-Cortés, T.; Pomar, F.; Espiñeira, J.M.; Merino, F.; Novo-Uzal, E. Purification and kinetic characterization of two peroxidases of Selaginella martensii Spring. involved in lignification. Plant Physiol. Biochem. 2012, 52, 130-139. [CrossRef]

21. Adams, J.B. Regeneration and kinetics of peroxidase inactivation. Food Chem. 1997, 60, 201-206. [CrossRef]

22. Petersen, T.N.; Brunak, S.; Von Hijne, G.; Nielsen, H. SIGNALP 4.0: Discriminating signal peptides from transmembrane regions. Nat. Methods 2011, 8, 785-786. [CrossRef]

23. Emanuelsson, O.; Nielsen, H.; Brunak, S.; Heijne, G.V. Predicting subcellular localization of proteins based on their N-terminal amino acid sequence. J. Mol. Biol. 2000, 300, 1005-1016. [CrossRef] 
24. Ligrone, R.; Ducket, J.G.; Renzaglia, K.S. Conducting tissues and phyletic relationships of bryophytes. Philos. Trans. R. Soc. Lond. B Biol. Sci. 2000, 355, 795-813. [CrossRef]

25. Ge, S.; Han, X.; Xu, X.; Shao, Y.; Zhu, Q.; Liu, Y.; Du, J.; Xu, J.; Zhang, S. WRKY15 suppresses tracheary element differentiation upstream of VND7 during xylem formation. Plant Cell 2020, 32, 2307-2324. [CrossRef]

26. Herrero, J.; Esteban-Carrasco, A.; Zapata, J.M. Looking for Arabidopsis thaliana peroxidases involved in lignin biosynthesis. Plant Physiol. Biochem. 2013, 67, 77-86. [CrossRef] [PubMed]

27. Ostergaard, L.; Teilum, K.; Mirza, O.; Mattsson, O.; Petersen, M.; Welinder, K.G.; Mundy, J.; Gajhede, M.; Henriksen, A. Arabidopsis ATP A2 peroxidase. Expression and high-resolution structure of a plant peroxidase with implications for lignification. Plant Mol. Biol. 2000, 44, 231-243. [CrossRef] [PubMed]

28. Kakrana, A.; Kumar, A.; Satheesh, V.; Abdin, M.Z.; Subramaniam, K.; Bhattacharya, R.C.; Srinivasan, R.; Sirohi, A.; Jain, P.K. Identification, validation and utilization of novel nematode-responsive root-specific promoters in Arabidopsis for inducing host-delivered RNAi mediated root-knot nematode resistance. Front. Plant Sci. 2017, 8, 2049. [CrossRef] [PubMed]

29. Kim, B.H.; Kim, S.Y.; Nam, K.H. Genes encoding plant-specific class III peroxidases are responsible for increased cold tolerance of the brassinosteroid-insensitive 1 mutant. Mol. Cells 2012, 34, 539-548. [CrossRef] [PubMed]

30. Fernández-Pérez, F.; Pomar, F.; Pedreño, M.A.; Novo-Uzal, E. The suppression of AtPrx52 affects fibers but not xylem lignification in Arabidopsis by altering the proportion of syringyl units. Physiol. Plant 2015, 154, 395-406. [CrossRef] [PubMed]

31. Irshad, M.; Canut, H.; Borderies, G.; Pont-Lezica, R.; Jamet, E. A new picture of cell wall protein dynamics in elongating cells of Arabidopsis thaliana: Confirmed actors and newcomers. BMC Plant Biol. 2008, 8, 94. [CrossRef]

32. Bindschedler, L.V.; Whitelegge, J.P.; Millar, D.J.; Bolwell, G.P. A two component chitin-binding protein from French bean: Association of a proline-rich protein with a cysteine-rich polypeptide. FEBS Lett. 2006, 580, 1541-1546. [CrossRef] [PubMed]

33. De Gara, L. Class III peroxidases and ascorbate metabolism in plants. Phytochem. Rev. 2004, 3, 195-205. [CrossRef]

34. Liszkay, A.; van der Zalm, E.; Schopfer, P. Production of reactive oxygen intermediates $\left(\mathrm{O}_{2}^{-}\right), \mathrm{H}_{2} \mathrm{O}_{2}$, and $\left.\mathrm{OH}\right)$ by maize roots and their role in wall loosening and elongation growth. Plant Physiol. 2004, 36, 3114-3123. [CrossRef]

35. Gazaryan, I.G.; Lagrimini, L.M.; Ashby, G.A.; Thorneley, N.F. Mechanism of indole-3-acetic acid oxidation by plant peroxidases: Anaerobic stopped-flow spectrophotometric studies on horseradish and tobacco peroxidases. Biochem. J. 1996, 313, 841-847. [CrossRef]

36. Hiraga, S.; Sasaki, K.; Ito, H.; Ohashi, Y.; Matsui, H. A large family of class III plant peroxidases. Plant Cell Physiol. 2001, 42, 462-468. [CrossRef]

37. Hatfield, R.D.; Rancour, D.M.; Marita, J.M. Grass cell walls: Story of cross-linking. Front. Plant Sci. 2017, 7, 2056. [CrossRef] [PubMed]

38. Fagerstedt, K.V.; Kukkola, E.M.; Koistinen, V.V.; Takahashi, J.; Marjamaa, K. Cell wall lignin is polymerised by class III secretable plant peroxidases in Norway spruce. J. Integr. Plant Biol. 2010, 52, 186-194. [CrossRef] [PubMed]

39. Ros Barceló, A.; Gómez Ros, L.V.; Esteban-Carrasco, A. Looking for syringyl peroxidases. Trends Plant Sci. 2007, 12, 486-491. [CrossRef] [PubMed]

40. Szklarczyk, D.; Gable, A.L.; Lyon, D.; Junge, A.; Wyder, S.; Huerta-Cepas, J.; Simonovic, M.; Doncheva, N.T.; Morris, J.H.; Bork, P.; et al. STRING v11: Protein-protein association networks with increased coverage, supporting functional discovery in genome-wide experimental datasets. Nucleic Acids Res. 2019, 47, D607-D613. [CrossRef]

41. Kim, S.J.; Kim, K.W.; Cho, M.H.; Franceschi, V.R.; Davin, L.D.; Lewis, N.G. Expression of cinnamyl alcohol dehydrogenases and their putative homologues during Arabidopsis thaliana growth and development: Lessons for database annotations? Phytochemistry 2007, 68, 1957-1974. [CrossRef] [PubMed]

42. Moinuddin, S.G.; Jourdes, M.; Laskar, D.D.; Ki, C.; Cardenas, C.L.; Kim, K.W.; Zhang, D.; Davin, L.B.; Lewis, N.G. Insights into lignin primary structure and deconstruction from Arabidopsis thaliana COMT (caffeic acid O-methyl transferase) mutant Atomt1 Org. Biomol. Chem. 2010, 8, 3928-3946. [CrossRef] [PubMed]

43. Møller, S.; Yi, X.; Velásquez, S.M.; Gille, S.; Hansen, P.L.M.; Poulsen, C.P.; Olsen, C.E.; Rejzek, M.; Parsons, H.; Yang, Z.; et al. Identification and evolution of a plant cell wall specific glycoprotein glycosyl transferase, ExAD. Sci. Rep. 2017, 7, 45341. [CrossRef] [PubMed]

44. Miyamoto, T.; Takada, R.; Tobimatsu, Y.; Suzuki, S.; Yamamura, M.; Osakabe, K.; Osakabe, Y.; Sakamoto, M.; Umezawa, T. Double knockout of OsWRKY36 and OsWRKY102 boosts lignification with altering culm morphology of rice. Plant Sci. 2020, $296,110466$. [CrossRef]

45. Martone, P.T.; Estevez, J.M.; Lu, F.; Ruel, K.; Denny, M.W.; Somerville, C.; Ralph, J. Discovery of lignin in seaweed reveals convergent evolution of cell-wall architecture. Curr. Biol. 2009, 19, 169-175. [CrossRef] [PubMed]

46. Koutaniemi, S.; Toikka, M.M.; Kärkönen, A.; Mustonen, M.; Lundell, T.; Simola, L.K.; Kilpeläinen, I.A.; Teeri, T.H. Characterization of basic $p$-coumaryl and coniferyl alcohol peroxidases from a lignin-forming Picea abies suspension culture. Plant Mol. Biol. 2005, 58, 141-157. [CrossRef] [PubMed]

47. Quiroga, M.; Guerrero, C.; Botella, M.A.; Barcelo, A.; Amaya, I.; Medina, M.I.; Alonso, F.J.; de Forchetti, S.M.; Tigier, H.; Valpuesta, V. A tomato peroxidase involved in the synthesis of lignin and suberin. Plant Physiol. 2000, 122, 1119-1127. [CrossRef]

48. Gabaldón, C.; López-Serrano, M.; Pedreño, M.A.; Ros Barceló, A. Cloning and molecular characterization of the basic peroxidase isoenzyme from Zinnia elegans, an enzyme involved in lignin biosynthesis. Plant Physiol. 2005, 139, 1138-1154. [CrossRef] [PubMed] 
49. Moural, T.W.; Lewis, K.M.; Barnaba, C.; Zhu, F.; Palmer, N.A.; Sarath, G.; Scully, E.D.; Jones, J.P.; Sattler, S.E.; Kang, C. Characterization of class III peroxidases from switchgrass. Plant Physiol. 2017, 173, 417-433. [CrossRef]

50. Cochrane, F.C.; Davin, L.B.; Lewis, N.G. The Arabidopsis phenylalanine ammonia lyase gene family: Kinetic characterization of the four PAL isoforms. Phytochemistry 2004, 65, 1557-1564. [CrossRef]

51. Baltas, M.; Lapeyre, C.; Bedos-Belval, F.; Maturano, M.; Saint-Aguet, P.; Roussel, L.; Duran, H.; Grima-Pettenati, J. Kinetic and inhibition studies of cinnamoyl-CoA reductase 1 from Arabidopsis thaliana. Plant Physiol. Biochem. 2005, 43, 746-753. [CrossRef] [PubMed]

52. Wang, J.P.; Naik, P.P.; Chen, H.C.; Shi, R.; Lin, C.Y.; Liu, J.; Shuford, C.M.; Li, Q.; Sun, Y.H.; Tunlaya-Anukit, S.; et al. Complete proteomic-based enzyme reaction and inhibition kinetics reveal how monolignol biosynthetic enzyme families affect metabolic flux and lignin in Populus trichocarpa. Plant Cell 2014, 26, 894-914. [CrossRef]

53. Solé-Gil, A.; Hernández-García, J.; López-Gresa, M.P.; Blázquez, M.A.; Agustí, J. Conservation of thermospermine synthase activity in vascular and non-vascular plants. Front. Plant Sci. 2019, 10, 663. [CrossRef]

54. Moghe, G.D.; Last, R.L. Something old, something new: Conserved enzymes and the evolution of novelty in plant specialized metabolism. Plant Physiol. 2015, 169, 1512-1523. [CrossRef]

55. Xu, Z.; Zhang, D.; Hu, J.; Zhou, X.; Ye, X.; Reichel, K.L.; Stewart, N.R.; Syrenne, R.D.; Yang, X.; Gao, P.; et al. Comparative genome analysis of lignin biosynthesis gene families across the plant kingdom. BMC Bioinform. 2009, 10 (Suppl. 11), S3. [CrossRef]

56. Labeeuw, L.; Martone, P.T.; Boucher, Y.; Case, R.J. Ancient origin of the biosynthesis of lignin precursors. Biol. Direct. 2015, 10, 23. [CrossRef] [PubMed]

57. Renault, H.; Werck-Reichhart, D.; Weng, J.K. Harnessing lignin evolution for biotechnological applications. Curr. Opin. Biotechnol. 2019, 56, 105-111. [CrossRef]

58. Benito, B.; Rodriguez Navarro, A. Molecular cloning and characterization of a sodium-pump ATPase of the moss Physcomitrella patens. Plant J. 2003, 36, 382-389. [CrossRef] [PubMed]

59. Kroemer, M.; Dreyer, M.K.; Wendt, K.U. APRV—A program for automated data processing, refinement and visualization. Acta Crystallogr. D Biol. Crystallogr. 2004, 60, 1679-1682. [CrossRef]

60. Minami, A.; Nagao, M.; Arakawa, K.; Fujikawa, S.; Takezawa, D. Abscisic acid-induced freezing tolerance in the moss Physcomitrella patens is accompanied by increased expression of stress-related genes. J. Plant Physiol. 2003, 160, 475-483. [CrossRef] [PubMed]

61. Richardt, S.; Timmerhaus, G.; Lang, D.; Qudeimat, E.; Corrêa, L.G.G.; Reski, R.; Rensing, S.A.; Frank, W. Microarray analysis of the moss Physcomitrella patens reveals evolutionarily conserved transcriptional regulation of salt stress and abscisic acid signaling. Plant Mol. Biol. 2010, 72, 27-45. [CrossRef] [PubMed]

62. Liu, N.; Zhong, N.Q.; Wang, Q.L.; Li, L.J.; Liu, X.L.; He, Y.K.; Xia, G.X. Cloning and functional characterization of PpDBF1 gene encoding a DRE-binding transcription factor from Physcomitrella patens. Planta 2007, 226, 827-838. [CrossRef]

63. de Marco, A.; Guzzardi, P.; Jamet, E. Isolation of tobacco isoperoxidases accumulated in cell-suspension culture medium and characterization of activities related to cell wall metabolism. Plant Physiol. 1999, 120, 371-382. [CrossRef] [PubMed]

64. Chomczynski, P. A reagent for the single-step simultaneous isolation of RNA, DNA and proteins from cell and tissue samples. BioTechniques 1993, 15, 532-534. [PubMed]

65. Pfaffl, M.W.; Horgan, G.W.; Dempfle, L. Relative expression software tool (REST) for group-wise comparison and statistical analysis of relative expression results in real-time PCR. Nucleic Acids Res. 2002, 30, e36. [CrossRef] [PubMed] 